\title{
Decreased Plasma Ascorbate Levels in Stage IV Melanoma Patients
}

Authors

Affiliations

\section{T. Schleich ${ }^{1}$, S. Rodemeister ${ }^{2}$, S. Venturelli ${ }^{3}$, T. Sinnberg ${ }^{4}$, C. Garbe ${ }^{4}$, C. Busch ${ }^{4}$}

${ }^{1}$ Department of Pediatrics, University of Tuebingen, Germany

${ }^{2}$ Institute of Biological Chemistry and Nutrition, University of Hohenheim, Germany

${ }^{3}$ Department of Internal Medicine I, University of Tuebingen, Germany

${ }^{4}$ Department of Dermatology, Section of Dermato-Oncology, University of Tuebingen, Germany

\author{
Key words \\ - vitamin C \\ - ascorbic acid \\ - cancer \\ - chemotherapy \\ ipilimumab \\ O scurvy
}

\begin{abstract}
$\nabla$

It has been reported that cancer patients frequently express low ascorbate (ascorbic acid, vitamin C) blood levels. However, so far this was not shown for melanoma patients.

Total ascorbate (TAA) levels were determined in plasma of healthy control individuals $(n=31$, mean age: 47.3 years, TAA: $64.86 \mu \mathrm{M}$ ) and in 126 melanoma patients (stage I: $\mathrm{n}=30$, mean age: 51 years, TAA: $59.95 \mu \mathrm{M}$; stage II: $\mathrm{n}=30$, mean age: 46.8 years, TAA: $58.85 \mu \mathrm{M}$; stage III: $\mathrm{n}=32$, mean age: 48.6 years, TAA: $57.27 \mu \mathrm{M}$; stage IV: $n=34$, mean age: 51.1 years, TAA: $47.16 \mu \mathrm{M})$. Plasma TAA levels in stage IV patients were significantly reduced by $27.3 \%$ when compared to healthy individuals $(p=0.0001$, t-test $)$. The reduced plasma TAA levels in stage IV patients negatively
\end{abstract}

\section{Introduction}

$\nabla$

An estimated 3.2 million new cases of cancer and 1.7 million deaths from cancer occurred in Europe in 2008 [1]. The fact that only $5-10 \%$ of all cancer cases are due to genetic defects and that the remaining $90-95 \%$ are due to lifestyle factors (including smoking, alcohol, physical inactivity, obesity and sun exposure), nutrition, infections and environmental pollutants provide major opportunities for preventing cancer [1]. Malignant melanoma is a tumor derived from melanocytes, manifesting mainly on the skin, and in rare cases, from mucous membranes. The tumor-specific 10-year survival for patients with primary melanomas without any evidence of metastasis is $75-85 \%$, decreasing to $20-40 \%$ for those patients with clinically apparent regional lymph node metastases. Distant organ metastases (stage IV disease) have a poor prognosis with a median survival in untreated patients of 6-9 months [2]. correlated with increased S100 and lactate dehydrogenase (LDH) levels. Further, plasma TAA levels were determined in additional 9 stage IV patients directly before and $24 \mathrm{~h}$ after intravenous polychemotherapy (carboplatin + paclitaxel, $\mathrm{n}=5$ ) or immunotherapy (ipilimumab, $\mathrm{n}=4$ ). TAA levels significantly decreased $24 \mathrm{~h}$ after therapy (mean TAA before therapy: $48.7 \mu \mathrm{M}$; mean TAA after therapy: $43.0 \mu \mathrm{M} ; 11.4 \%$ reduction, $\mathrm{p}<0.05$, t-test).

Ascorbate levels in the plasma of 126 melanoma patients were significantly decreased in the cohort of stage IV patients and were further decreased by polychemo- or immunotherapy in stage IV patients. Considering the importance of adequate ascorbate supply, ascorbate substitution in physiological doses could be considered for late-stage melanoma patients.

\section{Dr. med. Christian Busch} Department of Dermatology Section of Dermato-Oncology University of Tuebingen Liebermeisterstraße 20 72076 Tuebingen Germany Tel.: + 49/7071/2984555 Fax: + 49/7071/295 187 Christian.Busch@med. uni-tuebingen.de
Several studies have shown that plasma ascorbate levels are decreased in patients with malignancies, such as hepatocellular carcinoma [3], lung cancer [4], colorectal cancer [5] and prostate cancer [6].

A basic biochemical role for ascorbate is to accelerate hydroxylation reactions in a number of biosynthetic pathways. In many of these reactions, ascorbate directly or indirectly provides electrons to enzymes that require prosthetic metal ions in a reduced form to achieve full enzymatic activity. Ascorbate is required in the synthesis of carnitine from lysine, collagen and neurotransmitter synthesis, cytochrome P-450 activity, cholesterol metabolism, detoxification of exogenous compounds, and as an antioxidant [7]. Moreover, ascorbate is essential for immunoglobulin synthesis. In cell-mediated immunity, immunocompetence is exercised overwhelmingly by lymphocytes, which contain high concentrations of ascorbate relative to other cells. In addition, ascorbate is required for active phagocytosis and 
Table 1 Plasma total ascorbate levels of 126 stage I-IV melanoma patients and 31 controls.

\begin{tabular}{|c|c|c|c|c|c|}
\hline cohorts & $\mathbf{n}$ & mean age & TAA $[\mu \mathrm{M}]$ & $\%$ reduction in TAA & p-value, t-test \\
\hline control & 31 & 47.3 & 64.86 & & \\
\hline stage I & 30 & 51 & 59.95 & 7.6 & 0.253 \\
\hline stage II & 30 & 46.8 & 58.85 & 9.3 & 0.196 \\
\hline stage III & 32 & 48.6 & 57.27 & 11.7 & 0.069 \\
\hline stage IV & 34 & 51.1 & 47.16 & 27.3 & 0.0001 \\
\hline stage IV $S 100<0.1 \mu \mathrm{g} / \mathrm{I}$ & 12 & 52.8 & 51.78 & 20.2 & 0.028 \\
\hline stage IV S100>0.1 $\mathrm{gg} / \mathrm{I}$ & 21 & 50.9 & 44.04 & 32.1 & 0.0002 \\
\hline stage IV LDH<250 U/I & 21 & 51.8 & 52.05 & 19.8 & 0.006 \\
\hline stage IV LDH > $250 \mathrm{U} / \mathrm{I}$ & 12 & 51.1 & 37.76 & 41.8 & 0.0001 \\
\hline
\end{tabular}

TAA: total ascorbic acid; LDH: lactate dehydrogenase

enhances interferon production [7]. In line, impairment of lymphocyte activation, and macrophage and neutrophil mobilization were reported in scorbutic animals [9].

Aim of the study: Considering the importance of adequate ascorbate supply for hundreds of biochemical processes and a functional immune system, we asked whether plasma ascorbate levels correlated with disease stage in patients afflicted with malignant melanoma and whether intravenous systemic therapy (polychemotherapy or immunotherapy) in stage IV melanoma patients influenced the plasma ascorbate level.

\section{Materials and Methods}

$\nabla$

\section{Ethics statement}

The use of human plasma samples was approved by the medical ethical committee of the University of Tuebingen, Germany, and was performed in accordance with the Declaration of Helsinki Principles. The plasma samples were gathered during routine blood withdrawals. Every patient signed an informed consent form prior to blood withdrawal allowing the determination of plasma ascorbate level for research purposes.

\section{Recruitment of patients and controls}

Patients aged 18 years and older included in this study. Patients with artificial ascorbate supplementation (e.g. taking ascorbateor multi-vitamin pills) were excluded from this study. The control cohort consisted of healthy volunteering employees (nurses, dermatologists and laboratory staff) of the Department of Dermatology, University of Tuebingen, Germany, aged 18 years and older and was stratified by the exclusion criteria stated above. The control cohort and the stage I and stage II melanoma patients had an ECOG Performance Status grade 0 . The stage III melanoma patients had an ECOG Performance Status grade 0 or 1 , and the stage IV melanoma patients grade 3 or 4 .

\section{HPLC ascorbate measurements}

For the quantification of ascorbate in EDTA-plasma, samples were immediately mixed with an equal amount of $5 \%$ perchloric acid (Sigma, Munich, Germany) and stored at $-80^{\circ} \mathrm{C}$. The time between blood withdrawal and snap-freezing was less than $10 \mathrm{~min}$. For analysis of plasma ascorbate content, samples were thawed and centrifuged at $13000 \times \mathrm{g}$ for $5 \mathrm{~min}$ at $4^{\circ} \mathrm{C}$. For reduction of DHA and determination of TAA, the supernatant was mixed with $0.15 \mathrm{M}$ aqueous $\operatorname{Tris}(2$-carboxyethyl)phosphine (TCEP, ABCR, Karlsruhe, Germany) solution (v/v: 2/1) and incubated for $5 \mathrm{~min}$. Afterwards, the mixture was centrifuged at
$13000 \times \mathrm{g}$ for $5 \mathrm{~min}$ at $4^{\circ} \mathrm{C}$ and transferred into light-protected micro-vials for chromatographic analysis. As external standards, 2 aqueous ascorbic acid solutions ( $50 \mu \mathrm{M}$ and $100 \mu \mathrm{M})$ were prepared, diluted with an equal amount of perchloric acid (5\%), mixed with $0.15 \mathrm{M}$ aqueous TCEP solution (v/v: 2/1) incubated for $5 \mathrm{~min}$. The mixture was centrifuged at $13000 \times \mathrm{g}$ for $5 \mathrm{~min}$ at $4^{\circ} \mathrm{C}$ and transferred into light-protected micro-vials for chromatographic analysis. All analytical procedures were performed on ice and under dimmed light to avoid ascorbic acid degradation. For HPLC analysis, samples were placed in a cooled autosampler (Varian, Darmstadt, Germany). Chromatography was carried out on a reversed-phase column (Reprosil-Pur 120 C18 AQ $5 \mu \mathrm{M}$; Trentec, Gerlingen, Germany) with a mobile phase consisting of $5 \mathrm{mM}$ aqueous sodium phosphate buffer, $\mathrm{pH} 2.5$ at a flow rate of $1 \mathrm{ml} / \mathrm{min}$. Detection was performed by using an electrochemical detector (ESA Detektor Coulochem II, Chelmsford, United Kingdom) and a Model 5011 high-sensitivity analytical cell (ESA) at $-300 \mathrm{mV}$ (E1, upstream) and $+300 \mathrm{mV}$ (E2, downstream). Chromatograms were recorded and analyzed using Star chromatography workstation software version 5.31 (Varian, Darmstadt, Germany).

\section{Statistical analysis}

Statistical analysis of plasma ascorbate level was performed by using Student's t-test. P-values $<0.05$ were considered as statistically significant.

\section{Results}

$\nabla$

Plasma ascorbate is decreased in more advanced stages of melanoma and negatively correlates with plasma S100 and LDH levels

We determined total ascorbate (TAA) levels in plasma of healthy control individuals ( $\mathrm{n}=31$, mean age: 47.3 years, TAA: $64.86 \mu \mathrm{M}$ [26.68 $\mu \mathrm{M}-113.64 \mu \mathrm{M}$; SD: $1.11 \mu \mathrm{M}]$ ) and in 126 melanoma patients (stage I: $\mathrm{n}=30$, mean age: 51 years, TAA: $59.95 \mu \mathrm{M}$

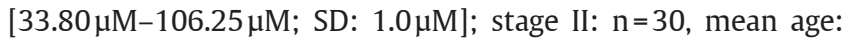
46.8 years, TAA: $58.85 \mu \mathrm{M}$ [28.83 $\mu \mathrm{M}-88.06 \mu \mathrm{M}$; SD: $1.20 \mu \mathrm{M}]$; stage III: $\mathrm{n}=32$, mean age: 48.6 years, TAA: $57.27 \mu \mathrm{M}$ [27.63 $\mu \mathrm{M}-80.11 \mu \mathrm{M}$; SD: $1.17 \mu \mathrm{M}]$; stage IV: $\mathrm{n}=34$, mean age: 51.1 years, TAA: $47.16 \mu \mathrm{M}[4.82 \mu \mathrm{M}-73.99 \mu \mathrm{M}$; SD: $1.0 \mu \mathrm{M}]$ ) by HPLC ( $\odot$ Table 1). Plasma TAA levels constantly decreased from stage I to stage III, but the difference was not significant when compared to the healthy controls. However, in stage IV patients the ascorbate plasma levels were significantly reduced by $27.3 \%$ when compared to healthy individuals $(p=0.0001$, $t$-test $)$. The ascorbate plasma levels of stage IV patients were also signifi- 
Table 2 Plasma total ascorbate levels before and after polychemo- and immunotherapy in stage IV melanoma patients.

\begin{tabular}{|c|c|c|c|c|c|}
\hline Patient & Treatment & TAA $[\mu \mathrm{M}]$ pre & TAA $[\mu \mathrm{M}]$ post & $\%$ reduction in TAA & p-value, t-test \\
\hline 1 & Carbotax & 41.1 & 36.2 & 11.9 & \\
\hline 2 & Carbotax & 8.5 & 6.9 & 18.1 & \\
\hline 3 & Carbotax & 53.9 & 46.0 & 14.7 & \\
\hline 4 & Carbotax & 64.3 & 45.4 & 29.4 & \\
\hline 5 & Carbotax & 46.9 & 42.2 & 10.1 & \\
\hline 1 & Ipilimumab & 74.0 & 64.4 & 13.0 & \\
\hline 2 & Ipilimumab & 56.4 & 51.6 & 8.4 & \\
\hline 3 & Ipilimumab & 52.3 & 48.8 & 6.6 & \\
\hline \multirow[t]{2}{*}{4} & Ipilimumab & 41.1 & 45.2 & -9.9 & \\
\hline & mean & 48.7 & 43.0 & 11.4 & $<0.05$ \\
\hline
\end{tabular}

TAA: total ascorbic acid; pre: before therapy; post: $24 \mathrm{~h}$ after therapy; Carbotax: carboplatin + paclitaxel

cantly lower than those of stages I-III patients $(\mathrm{p}=0.0006$ for stage I vs. stage IV; $\mathrm{p}=0.0041$ for stage II vs. stage IV; $\mathrm{p}=0.0044$ for stage III vs. stage IV; t-test) ( $\bullet$ Table 1 ). Plasma ascorbate levels negatively correlated with S100 and lactate dehydrogenase (LDH)-values (both prognostic markers for malignant melanoma) in stage IV patients: The lowest plasma ascorbate concentrations were measured in melanoma patients with pathologically elevated $(>0.1 \mu \mathrm{g} / \mathrm{l}) \quad$ S100-values (mean TAA: $44.04 \mu \mathrm{M}$ [4.82 $\mu \mathrm{M}-73.99 \mu \mathrm{M}$; SD: $0.94 \mu \mathrm{M}] ; 32.1 \%$ reduction compared to controls; $\mathrm{p}=0.0002$, t-test) and elevated ( $>250 \mathrm{U} / \mathrm{l}$ ) LDH-values

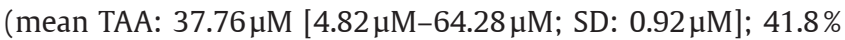
reduction compared to controls; $\mathrm{p}=0.0001$, t-test) ( $\bullet$ Table 1 ).

\section{Plasma ascorbate decreases after poly-chemotherapy and immunotherapy}

Second, we asked whether plasma ascorbate levels were influenced by systemic intravenous therapies performed on stage IV melanoma patients. To this end, plasma ascorbate levels were determined in 9 patients directly before and $24 \mathrm{~h}$ after either polychemotherapy (carboplatin + paclitaxel) or immunotherapy targeting CTLA4 (ipilimumab) $[9,10]$ ( $\diamond$ Table 2). We detected significantly reduced plasma ascorbate levels $24 \mathrm{~h}$ after therapy $(48.7 \pm 17.4 \mu \mathrm{M}$ vs. $43.0 \pm 14.7 \mu \mathrm{M} ; \mathrm{p}<0.05$, t-test) $(\odot$ Table 2$)$. We could not find a significant difference of the reduction of plasma ascorbate levels caused by poly-chemotherapy when compared to immunotherapy.

\section{Discussion \\ $\nabla$}

\section{Critical steps in the determination of plasma ascorbate} levels

In this study we show for the first time (to the best of our knowledge) that plasma ascorbate levels are reduced in stage IV melanoma patients when compared to healthy age-matched controls. Comparing the mean plasma ascorbate level of our healthy control cohort $(64.86 \mu \mathrm{M})$, we find a discrepancy in comparison to plasma ascorbate concentrations in the literature. In the European Prospective Investigation into Cancer and Nutrition (EPIC-Norfolk prospective study), the mean ascorbate serum concentrations in the different cohorts were 20.8$72.6 \mu \mathrm{M}$ with a calculated mean of $47.3 \mu \mathrm{M}$ (the exact mean was not communicated in the study) [11]. Secondly, in the more recent European Prospective Investigation into Cancer and Nutrition (EPIC-EURGAST), the mean ascorbate serum concentration in controls was $41.5 \mu \mathrm{M}$ [12]. However, in the latter 2 studies the processing of the blood samples for the determina- tion of ascorbate levels was not comparable to the conditions used in the present study. According to the methods section of the 2 studies, the authors did not enforce a quick handling and freezing of the blood samples. Considering the importance of a quick processing (stabilization and subsequent freezing in liquid nitrogen), which is indispensable due to the instability of ascorbic acid, and which we performed within the short time of less than 10 min after blood withdrawal for all samples, this obvious difference in sample handling most likely accounts for the difference of ascorbate concentrations on our control group when compared to the mean values of the latter 2 studies. In line with this assumption, in the second National Health and Nutrition Examination Survey, the median serum ascorbate concentrations were $49.4 \mu \mathrm{M}$ for men and $64.2 \mu \mathrm{M}$ for women [13]. In the latter study, the mean ascorbate concentration was comparable to our control group, and the blood samples were put on dry ice after stabilization in the mobile examination centers without further storage at $7^{\circ} \mathrm{C}$ over night. Thus, the quick stabilization and constant freezing (on dry ice) is comparable to the handling of the samples in our study and most likely accounts for the similar results obtained in the control samples. The lack of significant reduction of ascorbate plasma levels in stage I-III patients was obviously related to tumor burden. Stage I and II melanoma patients are completely tumor-free after resection of the primary melanoma, and stage III patients after adequate surgical treatment most of the times as well. Only in stage IV there is a metastatic tumor burden with all its consequences.

\section{Impact of high plasma ascorbate levels for cancer development}

Ascorbate-rich diets have protective effects on cancer development and other age-related diseases [14]. A recent meta-analysis of 8 case-control studies found a significant preventive effect on cervical neoplasms for increased intake of ascorbate [15]. Further, large epidemiological studies demonstrated a significantly increased risk of dying from cancer for adults [13] and for men [11] with low serum ascorbate concentrations. The prospective "European Prospective Investigation into Cancer and Nutrition" study revealed that higher plasma vitamin $\mathrm{C}$ levels were associated with a decreased risk of gastric cancer, independent of the particular gastric cancer anatomical subsite or histological subtype [12].

\section{Impact of low plasma ascorbate levels for cancer patients}

Previous clinical studies demonstrated reduced ascorbate levels in non-melanoma cancer patients $[4,16]$ sometimes even leading to scurvy [17]. In the latter case series the authors stressed the fact 
that scurvy should be considered in patients with cancer because of the high incidence of malnutrition caused by chemotherapy, cachexia caused by the disease, and other factors that might lead to an unbalanced dietary intake such as exclusive parenteral nutrition, depression, impaired taste, dysphagia, and abdominal pain. Further, weakness, anorexia, and depression are common in scurvy but also in patients with cancer. The authors concluded that clinicians should suspect ascorbate deficiency when a patient has haemorrhagic features without a clear explanation [17].

The importance of ascorbate for innumerable biochemical processes in the human organism is beyond dispute. Since humans and other primates lack the enzyme L-gulonolactone oxidase required for ascorbate synthesis, an adequate oral uptake is vital [7]. Considering the dependency of the immune system on adequate ascorbate supply [8] and the importance of the immune system for endogenous tumor control, and that, in addition, stage IV cancer patients frequently suffer from infections caused by therapy- or disease-induced neutrophilia, the additional ascorbate supplementation in physiological doses could be taken into consideration.

\section{Impact of anticancer therapies on plasma ascorbate levels}

Our data showed that stage IV melanoma patients are ascorbatedeficient, and that systemic therapies commonly used on such patients (chemotherapy, immunotherapy) further reduced the plasma ascorbate levels. However, the low number of patients in this cohort $(n=9)$ limits the generalizability of this finding to all stage IV melanoma patients undergoing systemic therapies in spite of its statistical significance. A similar therapeutic phenomenon was described by Marcus and colleagues, who demonstrated a severe ascorbate deficiency in 11 cancer patients (with metastatic melanoma, hypernephroma, and colon carcinoma), occurring as the result of adoptive immunotherapy with highdose interleukin 2 and lymphokine-activated killer cells [8]. Considering that so far no study has shown negative effects of ascorbate supplementation in cancer patients, our results suggest that especially late-stage melanoma patients (stage IV) might benefit from ascorbate supplementation.

\section{Potential anticancer mechanisms of ascorbate on melanoma cells}

A number of recent pre-clinical reports have demonstrated a decreased melanoma growth (both after s.c. and intraperitoneal injection) in mice unable to synthesize ascorbic acid (gulonolactone oxidase knockout mice) upon ascorbate supplementation $[18,19]$. In the latter 2 studies, ascorbate supplementation was accompanied by decreased serum pro-inflammatory cytokine (IL-6, IL-1 $1 \beta$ ) levels as well as enhanced encapsulation of tumors. IL-6, secreted by melanoma cells, promotes melanoma growth by inhibition of apoptosis and induces tumor angiogenesis. Further, an increased serum concentration of IL-6 correlates with a worse prognosis in melanoma patients [20]. This obvious impact of ascorbate on the immune response is in line with its crucial role in immunoglobulin synthesis. Moreover, lymphocytes contain high concentrations of ascorbate, and ascorbate is required for phagocytosis and enhances interferon production [7]. In addition, scorbutic animals show an impairment of lymphocyte activation and macrophage and neutrophil mobilization [8]. Together, for the immune response, ascorbate seems to be a double-edged sword functioning both as pro- and anti-inflamma- tory stimulus that inhibits tumor growth. On the cellular level, growth inhibition of melanoma cells was driven by cell cycle arrest and caspase-3 activation with subsequent apoptosis induction [21]. Further, ascorbate reduced the expression of the transferrin receptor in melanoma cells, resulting in a down-regulation of cellular iron uptake followed by apoptosis induction [22]. Due to recent pharmacokinetic data showing that intravenous administration of ascorbate results in high plasma levels not achievable by oral uptake and in a significant flux of $\mathrm{H}_{2} \mathrm{O}_{2}$ to tumors, the use of i.v. ascorbate in pharmacological doses in the treatment of cancer seems plausible and is currently being investigated in clinical trials and in experimental studies [23].

\section{Conclusions \\ $\nabla$}

We were able to demonstrate that plasma ascorbate levels were significantly lower in stage IV melanoma patients. Systemic therapy on stage IV patients further reduced plasma ascorbate levels. Considering the importance of adequate ascorbate supply for biochemical processes and host defense, our results suggest that late-stage melanoma patients might benefit from physiological ascorbate supplementation.

\section{Acknowledgements}

$\nabla$

This project was supported by grants from the Deutsche Forschungsgemeinschaft SFB 773: "Understanding and overcoming drug resistance of solid tumors" to CG and CB. SV was supported by a research fellowship from the Deutsche Forschungsgemeinschaft (VE671/2-1). CB and SV received a research grant from the Wissenschaftsfoerderung der Deutschen Brauwirtschaft e.V.

\section{References}

1 Gonzalez CA, Riboli E. Diet and cancer prevention: Contributions from the European Prospective Investigation into Cancer and Nutrition (EPIC) study. Eur J Cancer 2010; 46: 2555-2562

2 Garbe C, Peris K, Hauschild A, Saiag P, Middleton M, Spatz A, Grob JJ, Malvehy J, Newton-Bishop J, Stratigos A, Pehamberger H, Eggermont A. Diagnosis and treatment of melanoma: European consensus-based interdisciplinary guideline. Eur J Cancer 2010; 46: 270-283

3 Yamamoto Y, Yamashita S, Fujisawa A, Kokura S, Yoshikawa T. Oxidative stress in patients with hepatitis, cirrhosis, and hepatoma evaluated by plasma antioxidants. Biochem Biophys Res Commun 1998; 247: 166-170

4 Esme H, Cemek M, Sezer M, Saglam H, Demir A, Melek H, Unlu M. High levels of oxidative stress in patients with advanced lung cancer. Respirology 2008; 13: 112-116

5 Bhagat SS, Ghone RA, Suryakar AN, Hundekar PS. Lipid peroxidation and antioxidant vitamin status in colorectal cancer patients. Indian J Physiol Pharmacol 2011; 55: 72-76

6 Akinloye 0 , Adaramoye 0 , Kareem 0 . Changes in antioxidant status and lipid peroxidation in Nigerian patients with prostate carcinoma. Pol Arch Med Wewn 2009; 119: 526-532

7 González MJ, Miranda-Massari JR, Mora EM, Guzmán A, Riordan NH, Riordan HD, Casciari JJ, Jackson JA, Román-Franco A. Orthomolecular oncology review: ascorbic acid and cancer 25 years later. Integr Cancer Ther 2005; 4: 32-44

8 Marcus SL, Dutcher JP, Paietta E, Ciobanu N, Strauman J, Wiernik PH, Hutner SH, Frank O, Baker H. Severe hypovitaminosis Coccurring as the result of adoptive immunotherapy with high-dose interleukin 2 and lymphokine-activated killer cells. Cancer Res 1987; 47: 4208-4212

9 Pflugfelder A, Eigentler TK, Keim U, Weide B, Leiter U, Ikenberg K, Berneburg $M$, Garbe C. Effectiveness of carboplatin and paclitaxel as firstand second-line treatment in 61 patients with metastatic melanoma. PLOS One 2011; 6: e16882 
10 Hodi FS, O'Day SJ, McDermott DF, Weber RW, Sosman JA, Haanen JB, Gonzalez R, Robert C, Schadendorf D, Hassel JC, Akerley W, van den Eertwegh AJ, Lutzky J, Lorigan P, Vaubel JM, Linette GP, Hogg D, Ottensmeier CH, Lebbé C, Peschel C, Quirt I, Clark JI, Wolchok JD, Weber JS, Tian J, Yellin MJ, Nichol GM, Hoos A, Urba WJ. Improved survival with ipilimumab in patients with metastatic melanoma. N Engl J Med 2010; 363: 711-723

11 Khaw KT, Bingham S, Welch A, Luben R, Wareham N, Oakes S, Day $N$. Relation between plasma ascorbic acid and mortality in men and women in EPIC-Norfolk prospective study: a prospective population study. European Prospective Investigation into Cancer and Nutrition. Lancet 2001; 357: 657-663

12 Jenab $M$, Riboli E, Ferrari P, Sabate J, Slimani N, Norat T, Friesen $M$, Tjønneland A, Olsen A, Overvad K, Boutron-Ruault MC, Clavel-Chapelon F, Touvier M, Boeing H, Schulz M, Linseisen J, Nagel G, Trichopoulou A, Naska A, Oikonomou E, Krogh V, Panico S, Masala G, Sacerdote C, Tumino $R$, Peeters PH, Numans ME, Bueno-de-Mesquita HB, Büchner FL, Lund E, Pera G, Sanchez CN, Sánchez MJ, Arriola L, Barricarte A, Quirós JR, Hallmans G, Stenling R, Berglund G, Bingham S, Khaw KT, Key T, Allen $N$, Carneiro F, Mahlke U, Del Giudice G, Palli D, Kaaks R, Gonzalez CA. Plasma and dietary vitamin $C$ levels and risk of gastric cancer in the European Prospective Investigation into Cancer and Nutrition (EPICEURGAST). Carcinogenesis 2006; 27: 2250-2257

13 Loria CM, Klag MJ, Caulfield LE, Whelton PK. Vitamin C status and mortality in US adults. Am J Clin Nutr 2000; 72: 139-145

14 Polidori MC. Antioxidant Micronutrients in the Prevention of Agerelated Diseases. J Postgrad Med 2003; 49: 229-235
15 Myung SK, Ju W, Kim SC, Kim H. Korean Meta-analysis (KORMA) Study Group. Vitamin or antioxidant intake (or serum level) and risk of cervical neoplasm: a meta-analysis. BJOG 2011; 118: 1285-1291

16 Grober U. Vitamin C in complementary oncology - update 2009. Med Monatsschr Pharm 2009; 32: 263-267

17 Fain O, Mathieu E, Thomas M. Scurvy in patients with cancer. BMJ 1998; 316: 1661-1662

18 Cha J, Roomi MW, Ivanov V, Kalinovsky T, Niedzwiecki A, Rath M. Ascorbate supplementation inhibits growth and metastasis of B16FO melanoma and 4T1 breast cancer cells in vitamin C-deficient mice. Int J Oncol 2013; 42: 55-64

19 Cha J, Roomi MW, Ivanov V, Kalinovsky T, Niedzwiecki A, Rath M. Ascorbate depletion increases growth and metastasis of melanoma cells in vitamin C deficient mice. Exp Oncol 2011; 33: 226-230

20 Hoejberg L, Bastholt L, Schmidt H. Interleukin-6 and melanoma. Melanoma Res 2012; 22: 327-333

21 Lin SY, Lai WW, Chou CC, Kuo HM, Li TM, Chung JG, Yang JH. Sodium ascorbate inhibits growth via the induction of cell cycle arrest and apoptosis in human malignant melanoma A375.S2 cells. Melanoma Res 2006; 16: 509-519

22 Kang JS, Cho D, Kim YI, Hahm E, Kim YS, Jin SN, Kim HN, Kim D, Hur $D$, Park H, Hwang YI, Lee WJ. Sodium ascorbate (vitamin C) induces apoptosis in melanoma cells via the down-regulation of transferrin receptor dependent iron uptake. J Cell Physiol 2005; 204: 192-197

$23 \mathrm{Du}$ J, Cullen JJ, Buettner GR.. Ascorbic acid: chemistry, biology and the treatment of cancer. Biochim Biophys Acta 2012; 1826: 443-457 